\title{
Discordance and concordance on perception of quality care at end of life between older patients, caregivers and clinicians: a scoping review
}

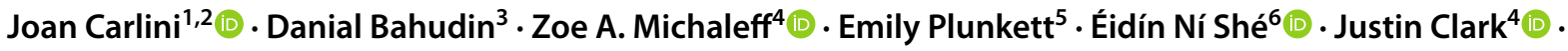 \\ Magnolia Cardona ${ }^{4,7}$ (D)
}

Received: 18 April 2021 / Accepted: 26 July 2021 / Published online: 12 August 2021

(c) European Geriatric Medicine Society 2021

\section{Key summary points}

Aim The purpose of this review was to synthesise and critically analyse the points of discordance and concordance between consumers and clinicians about quality end-of-life care.

Findings Respecting patient autonomy and the role of family is now clearly understood, but the role of healthcare provider and purpose of treatment withdrawal are still a matter of conflict between clinicians and patients or families. Clinician awareness of the possibility of misunderstanding by patients or families is the first step in preventing discordance of views on care quality at the end of life.

Message To prevent the conflict between parties, further efforts are needed to investigate ways to better communicate the goals of care, limitations of treatment and uncertainty associated with forecasting time to end of life.

\begin{abstract}
Background This scoping review aimed to investigate the presence of discordance or concordance in the perceptions of end-of-life (EOL) care quality between consumers (i.e. patients aged over 60 in their last years of life and/or their informal caregivers) and clinicians, to inform further improvements in end-of-life care service delivery.

Methods A scoping review of qualitative and quantitative studies was systematically undertaken by searching for English language publications in MEDLINE database and manual reference search of eligible articles. Thematic analysis was employed to identify and extract common concordance and discordance themes leading to the development of analytical constructs. Articles were eligible for inclusion if they reported on consumers' (i.e. older patients aged $60+$ years in their final years of life and/or their informal caregivers) and clinicians' (doctors, nurses, social workers, etc.) perspectives on quality of medical, surgical or palliative/supportive care administered to older adults in the last year of life across all healthcare settings.
\end{abstract}

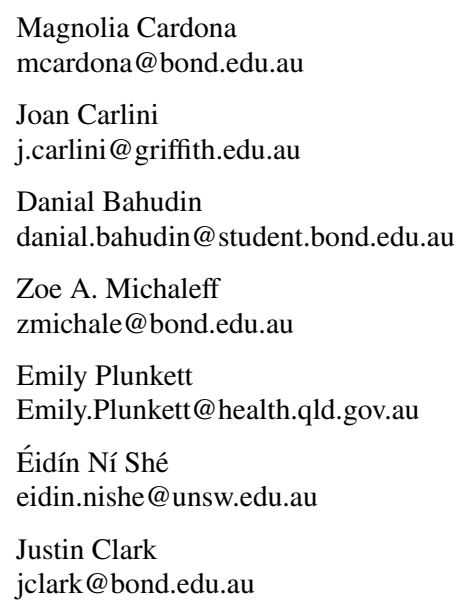

1 School of Business, Griffith University, Southport, QLD, Australia

2 Gold Coast University Hospital Consumer Advisory Group, Southport, QLD, Australia

3 Faculty of Health Sciences and Medicine, Bond University, Robina, QLD, Australia

4 Institute for Evidence Based Healthcare, Bond University, Robina, QLD, Australia

5 Palliative Care Service, Robina Hospital, Robina, QLD, Australia

6 School of Population Health, University of New South Wales, Kensington, NSW, Australia

7 Evidence Based Practice Professorial Unit, Gold Coast University Hospital, Level 2, PED building, 1 Hospital Boulevard, Southport, QLD 4215, Australia 
Results Of the 2736 articles screened, 21 articles were included. Four themes identified concordance between consumers' and clinicians' perceptions of care quality: holistic patient care; coordinated care that facilitated EOL; the role of family at EOL; and impact of prognostic uncertainty on care planning. Three themes emerged for discordance of perceptions: understanding the patient needs at EOL; capacity of healthcare system/providers to accommodate family needs; and knowledge and communication of active or palliative care at EOL.

Conclusions While progress has been made on promoting patient autonomy and respecting the family role in representing patient's best interest, gaps remain in terms of care coordination, communication of prognosis, public understanding of the meaning of goals of care including de-escalation of management and enactment of advance care directives by clinicians for people with diminished decision capacity. Public understanding of the meaning of "comfort" care and the need to prevent over-treatment are essential for their satisfaction with care and their ability to embrace the concept of a good death.

Keywords Caregivers · End-of-life care · Family conflict · Older adults · Professional-patient relations · Professionalfamily relations $\cdot$ Quality of health care

\section{Introduction}

End-of-life (EOL) care is defined as "physical, spiritual and psychosocial assessment, care and treatment delivered by health professionals and ancillary staff" [1] but also integrates requirements for global public health capacity and appropriate information strategies [2]. Dying trajectory in the last year of life for older people ( $\geq 65$ years) with chronic multimorbidity such as heart failure, kidney failure, lung disease, frailty or malignancies involves two distinct phases: periods of exacerbated illness that may be reversible and a shorter timeframe involving days to weeks where clinical deterioration is unlikely to be reversed. End-of-life care for older people is provided across many healthcare settings, including in people's homes, aged care and nursing homes, but most care still occurs in acute hospitals [3, 4]. The need to provide support for EOL care is gaining importance as the world population is ageing, with projections that the proportion of those aged $60+$ years is estimated to double and the proportion of $80+$ to triple in the next three decades [5]. Ideally, EOL care discussions would encompass emphasis on mitigation of suffering through avoidance of hospitalisations if appropriate [6] and pathways to achieve preferred place of death [7].

Perceptions of what constitutes good quality care at EOL include appropriate patient management and preferred place of death and are known to vary from the providers [8] to the patient receiving it and the perspective of their family [9]. The emotional burden and challenge of communicating poor prognosis to patients, who are either critically ill or suffer from irreversible chronic illness, and their family are well recognised in the literature [10]. Difficulties in achieving a common understanding stem from many angles, such as over-optimistic perception of risk of death among surrogate decision-makers underestimating the true risk [11], patients' unwillingness or lack of readiness to discuss the potential outcome of death [12], and the clinicians' delays in recognition of poor prognosis and need for transitioning patients from active treatment to palliative care management [13]. While intensive care clinicians may follow consensusbased guidelines on withholding or withdrawing life-sustaining treatments to enable optimal end-of-life care quality [14], families face the dilemma of having to decide on, or agree with, clinicians suspending treatment for older patients to prevent harm or prolonged suffering. Making these distressing judgments about whether and when to discontinue active treatments has moral, religious and legal implications [15-17]. Consequently, disagreements on perceived appropriateness of quality of end-of-life care provided in the last months of life are commonly reported [18, 19]. Patients' and their families' satisfaction with terminal care is known to be associated with non-clinical aspects such as achieving the preferred place of death, knowing whether their relative is "sick enough to die" [20] and receiving emotional or spiritual support [21]. To date, the perspectives and preferences of end-of-life care quality between consumers (patients/family caregivers or significant others) and clinicians derived from primary studies have not been synthesised to identify areas of concordance and discordance.

This scoping review aimed to synthesise and critically analyse the points of discordance and concordance between consumers and clinician about quality end-of-life care. The specific objectives of this review were to:

1. Identify the main themes of concordance on quality endof-life care between clinicians and healthcare consumers (patients, families and informal caregivers).

2. Identify the main themes of discordance on quality endof-life care between clinicians and healthcare consumers (patients, families and informal caregivers).

This work is now more relevant as the COVID-19 pandemic has highlighted key challenges in providing inclusive holistic end-of-life care for older people [22, 23]. The 
findings of this study may further contribute to informing strategies to improve communication on and delivery of terminal care for older people.

\section{Materials and methods}

\section{Design and data sources}

This work is a subset reporting of a larger review with protocol available on Open Science Framework (https://osf.io/ 5u964/). This scoping review used the ENTREQ (Enhancing transparency in reporting the synthesis of qualitative research) framework [24], developed to increase research reliability. This method bridges the gap between traditional quantitative and narrative reviews, as it provides a synthesis of both qualitative and quantitative health researches. A scoping review is a knowledge synthesis methodology that is intended to review evidence addressing broad topics inclusive of many different study designs [25]. In this scoping review, a systematic review approach was used to identify included studies. Thematic analysis was adopted for data synthesis.

\section{Search strategy}

MEDLINE was searched from inception to May 2019 by the information specialist in our team (JC2) using the systematic review accelerator tools (http://sr-accelerator.com/\#/) [26]. The search strategy included both MESH terms and keywords related to "terminal care" and the perspectives of both "older patients", "surrogates" "carers" and "clinicians. The complete search strategy can be viewed in Supplement 1, Table S1.1. The electronic database search was supplemented with manual reference list searches from both the included studies and other systematic reviews related to but not eligible for inclusion in our study. English language and time restrictions (January 2010-June 2019) were applied to ensure relevance to today's healthcare practice. Attempts to contact article authors were made when full text was unclear about eligibility criteria.

\section{Study selection and eligibility criteria}

Inclusion criteria comprised: (1) qualitative and quantitative studies of any research design; (2) included both consumers, healthcare professionals' perspective; and (3) reported on the opinions, perceptions or perspectives on what constituted quality EOL care related to any aspect of the medical, surgical and palliative/supportive care delivered to an older adult $(60+$ years) in their final years of life (not including acute injuries). For the purpose of this review, only studies that reported the perspective of both consumers and healthcare professionals in the one article were eligible for inclusion. This criterion ensured any concordant and/ or discordant themes identified reflected peoples' experiences of the same situation and setting and not differences between healthcare professionals or settings. "End of life" was defined either by the authors of an eligible article or using the CriSTAL (Criteria for Screening and Triaging to Appropriate aLternative care) tool, an objective checklist previously validated for identification of risk of short-term death in older patients [27]. We excluded articles if all eligibility criteria could not be conclusively identified (e.g. palliative care without specifying age of participants; or older people but unclear whether they were at risk of death within the year, and whether perspective from only one of the target groups was included). Paired authors (DB and ZAM, or MC) conducted a title and abstract screening of the total citations obtained against the eligibility criteria. Discrepancies were resolved through group discussions between these four reviewers. Full-text reviews were conducted by paired reviewers (DB and ZAM).

\section{Data extraction and synthesis}

Two reviewers (DB and ZAM) independently extracted the data using a template including study aims, location, year of publication, study design, participants and perspectives of both consumers and healthcare professionals on the quality of end-of-life care provided to older adults in their final years of life. After multiple readings of the eligible papers, DB and ZAM manually extracted relevant findings and organised them into codes using NVivo software. Using line-by-line coding, the findings of the primary studies were descriptively coded (DB), before developing analytical themes. During the first round, the coding rule used was to capture data from each paper from both (1) clinician perspective and (2) consumer perspective. In the second stage, coded data were reviewed to identify the emergence of themes. At regular intervals, inter-coder reliability checks were taken to ensure that the data were coded consistently with the coding rules. Four other reviewers (MC, ENS, EP and JC, a health service consumer with qualitative research skills) independently read and reviewed the thematic analysis to confirm reliability and comprehensiveness. We used inter-coder reliability, a methodology to check and control analytical interpretations which required unanimous author agreement to finalise a concept [28]. We achieved this by providing researchers (DB, ZAM, MC, JC) with a description of initial set of conception, including first-round coded data. The researchers then were required to explain and justify each set. Thus, a rigorous check of interpretation of raw data and definitions provided general agreement, and was verified by ENS and EP. The synthesis process, the method employed, was adapted from Thomas and Harden's thematic analysis 
technique [29]. During the entire process, the original data were kept with the analysis to ensure that the original meanings were conserved, and the judgement and insights of the reviewers were of the original data. Through an iterative process, the analytical constructs for both concordance and discordance perspectives were finalised as a group.

\section{Results}

The initial search from the databases yielded a total of 2736 citations. After title and abstract screening (DB and ZAM), a further 2597 were removed. After a full-text assessment, a total of 21 articles were eligible for inclusion in this review (Fig. 1).

\section{Study characteristics}

Twenty-one studies met all eligibility criteria: 10 qualitative studies, 9 quantitative and 2 mixed studies. Included studies reported on the perspectives of 2116 participants in

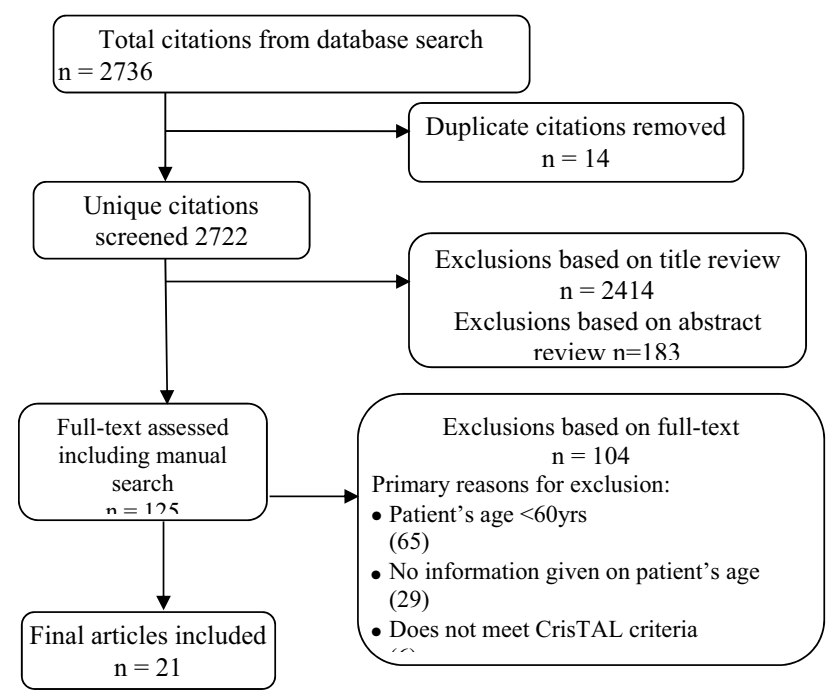

Fig. 1 Flow diagram of screening and article selection

10 countries: UK (6), USA (5), other Europe (6), Australia (2), Canada (1) Hong Kong (1) (Table 1). The qualitative study designs had sample sizes ranging from 34 to 125 , and median 60, IQR 47-95; the corresponding values for quantitative studies were range 20-520, median 51, IQR 33-127. Almost half of the included studies (10/21 eligible) were cross-sectional surveys [30-38] and nine used qualitative interviews or focus groups [39-48], with two using both methods $[49,50]$ : only one used retrospective record review [40] and two used cohort designs [44, 51] (Table 1). Approximately half of the included studies $(11 / 21)$ covered the perspectives of all three target groups of healthcare providers, patients and informal caregivers, with almost (8/21) reporting the perspective of informal caregivers rather than patients either were deceased or had dementia. Several studies included multiple settings. The most common settings covered were long-term care facilities (11) followed by hospitals (8), community including general practice (8), and hospices or other palliative care service (4).

\section{Thematic synthesis of results}

A total of four concordance and three discordance themes were identified from the data. A summary of the key themes and sub-themes is shown in Fig. 2.

\section{Concordance perspectives}

Four main concordance themes emerged from the data: holistic patient-centred care, coordinated healthcare system to facilitate end-of-life care, the role of the family at the end of life, and impact of prognostic uncertainty on care planning.

\section{Holistic patient-centred care at the end of life}

"Even if the doctors want the best for you, it is not always possible, so I brought that up with the physician. Now it is my quality of life not theirs, irrespective of how well they mean" (patient) [34].

"If you are at a hospital or residential home, then the room or apartment is the patient's. You are the guest. You knock on the door before you enter" (Nurse) [34].

Patient-centred care is defined as "providing care that is respectful of and responsive to individual patient preferences, needs, and values, and ensuring that patient values guide all clinical decisions" [52]. At the core of this theme was ensuring that a patient's individuality and preferences guide care delivery [32, 34, 35, 37, 41, 43, 45, 47, 48]. Much of the coverage noted the need for staff to see beyond a patient's diagnosis and treat them as an individual and that this is promoted in circumstances where a continuity of care is available [43]. In one study, clinicians' descriptions of a good death included where a personal relationship with patients, built over successive exchanges, existed [47].

The ability to empathise was described positively by both parties because it enables staff to understand the needs of residents and their families and supports active listening and reciprocal communication, thus ensuring greater dignity and respect for the patient at end of life [32, 34]. Enabling a patient's communication via formal or informal mechanisms around their will and preference was also stressed [34, 47], and so too was understanding cultural differences. For example, in Chinese nursing homes where death for many 


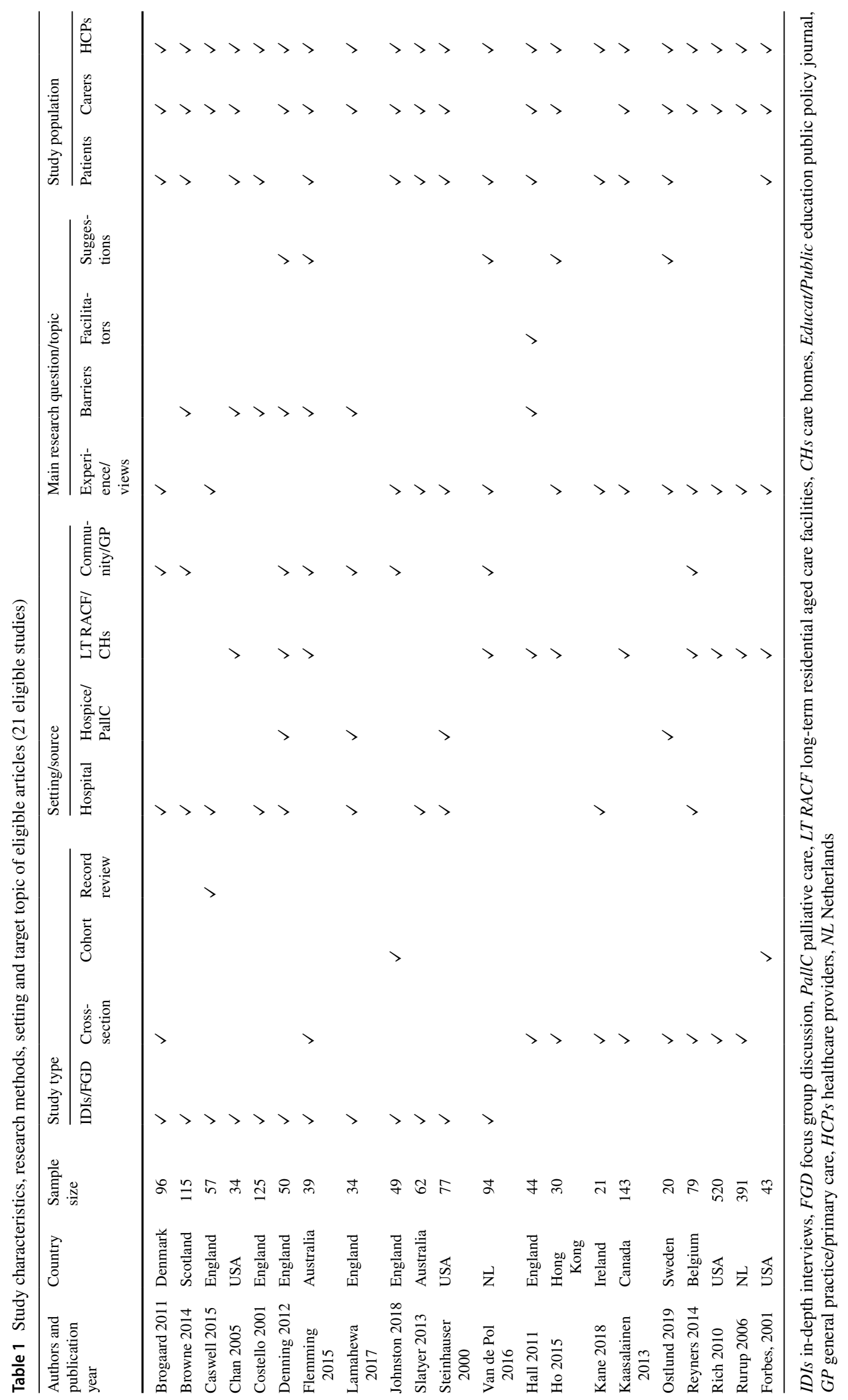


residents was a "bad omen", meant patients did not wish to discuss it openly [41]. The end-of life care setting [35, 43] and physical environment (such as the availability of private spaces $[34,50]$ ) were noted as central to patient-centred care. For example, hospitalisation impacted negatively on end-of-life care for people with dementia, and hospice care was stressed as the preferred location for end of life by residents and their family carers in 13 US nursing homes [51].

In addition, descriptions emerged including encouraging patients to take control of their own care [34, 47]. Having control over their own care planning and activities at the end of life was stressed by both consumers and various clinicians including doctors and nursing staff. Attention to the psychosocial $[33,34,42,47]$ and spiritual needs of the person [34, 47] was highlighted in a study on EOL care for people with chronic heart failure [33]. Patients spoke of the anxiety they and their caregivers experienced and advocated that psychological distress should be frequently assessed and discussed with them. Another article suggested clinicians can help support and prepare patients for death spiritually: enabling a life review, saying goodbye and resolving any identified conflicts for patients [47]. Sensitive patient-centred communication was stressed in many descriptions of holistic patient-centred care [32-34]. A need for sensitivity towards patients who had difficulty accepting that they cannot manage their activities of daily living was emphasised in an article containing suggestions from older patients, significant others and clinician's of how to conserve dignity in palliative care [34].

\section{Co-ordinated healthcare systems to facilitate end-of-life care}

"From the very start of clinical admission, our entire team of doctors, nurses and social workers would go through the patient medical records together, identify his or her needs as well as those of the family, and coordinate care that best reflect their wishes." (Medical professional) [32].

"I think the consistency of care a patient receives is very important .... It is wonderful that my wife can receive all the care that she needs with the same care team from the very beginning till her very last days." (family caregiver) [32].

The provision of contemporary care that is coordinated and consistent between healthcare providers and delivered by a core team of skilled professionals was considered to alleviate uncertainty and vulnerability at end of life. When end-of-life pathways encouraged continuity and coordinated care [31, 32, 35, 39-41, 44, 46, 48], there was evidence that patients had more options about the location of death, better coordination between providers across boundaries and increased multidisciplinary teamwork [44]. But when it came to coordinated care, several inefficiencies were highlighted including turnover of nurses and GPs, flawed communication due to the lack of familiarity with each other,
Fig. 2 Summary of the main themes and sub-themes $(n=21$ studies). EOL end of life
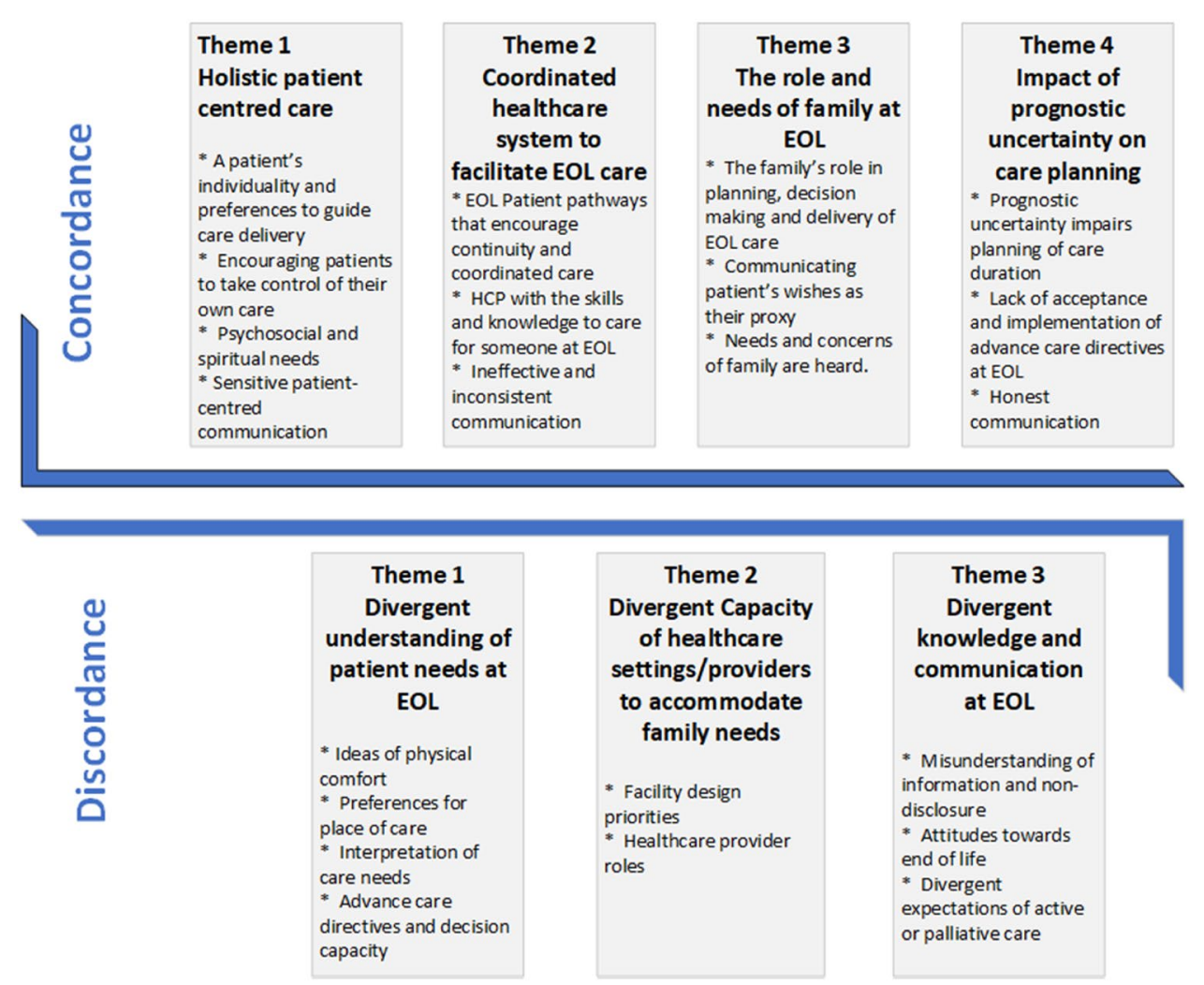
poor role definition and suboptimal recording of treatment plans [48]. Ensuring health and social care professionals had the relevant skills and knowledge to care for a person at end of life [31, 34, 43, 48] including time and space to attend training was stressed. A UK study [31] found that it was care assistants who provided much of the day-to-day care to residents in the care homes and that they were often excluded from training and reflection sessions. A lack of coordinated care was also apparent in one study where clinicians were uncertain about whose responsibility it was to control symptoms [30]. Ensuring effective and consistent communication $[34,35,40,46,48]$ was highlighted as important; for example, the experience of carers was enhanced when adequate clinician time was given to improve carer understanding [40] although challenges to achieving this were identified, e.g. obtaining information from hospital staff when staff had very little time to sit with families.

\section{The role and needs of family at end of life}

“... and I feel comfortable telling them about my wishes and concerns. I think this form of partnership is very important. It makes me feel dignified because they value my opinions and take the time to address my needs. (family caregiver) [32].

"Family members play a critical role in the overall planning and delivery of end of life care, we involve them as much as we can in the care decision making process. We hold regular care conferences.." (health professional) [32].

The family are a critical conduit at the end of a person's life. Family/significant others are a source of information, care and support. Providing this care is physically, emotionally and socially demanding, and the literature advocates that the "carers" too need care and support. Healthcare providers' understanding of the role of families in planning, making care decisions and delivering end-of-life care [32, 34] was repeatedly identified as important. In a study examining the system dynamics necessary for successfully implementing a novel end-of-life integrated care pathway among terminally ill Chinese nursing home residents, the individual resident and their family were considered as one unit of care and were integral to providing care at the end of life [32]. Several articles [32, 34, 45] noted a role for families communicating a patients wishes as their proxy in end-oflife scenarios. In particular, for people with dementia [45], healthcare workers recognised that family members had intimate knowledge of their patients' values, preferences, likes and dislikes. The need for recognition of the specific needs of family members $[31,34,43]$ was highlighted with several articles describing the burden associated with the caring role and how this affected family members both physically and emotionally. Family members advocated the need for greater access to psychosocial and bereavement supports, especially when preparing families for the aftermath of the death of a patient.

\section{Impact of prognostic uncertainty on care planning}

"I think it seems to me not like cancer where they say you've got five months to live or you've got a year but nobody has said that." (Patient) [39].

"It is difficult to predict when a resident is going to diethose who you expect will return following an admission, do not and those that you think you will not see again, come back." (Care Home Manager) [43].

Not knowing the time to death makes planning for duration of end-of-life care difficult for all involved; however, acceptance and implementation of Advance Care Plans can aid such preparation. The impact of prognostic uncertainty on care planning $[31,43,45,46,51]$ was frequently described. Confusion may arise between family carers and clinicians around timeframes as both groups described uncertainty knowing when a person with dementia was entering the terminal phase of the illness and that this could lead to inappropriate or reactive care [43]. More experienced end-of-life staff who were more prepared in managing this lack of clarity was ideal [36]. As to a lack of acceptance and implementation of advance care directives at the end of life of a person [31, 36, 37, 43, 45, 47, 48], we found that for care home staff, advance care planning helped prevent confusion when residents were rushed to the acute hospital and even protected the nursing home if they did not take a resident to hospital as outlined in their plan [31]. Further, healthcare staff accepted the importance of advance directives for people with dementia, in particular when the person is at the end stage and refuses to eat and drink [37]. The importance of honest communication was conversed in articles $[34,39]$ including honest disclosure by medical professionals of diagnostic, prognostic and treatment information along with patient and then honestly communicating these circumstances to family caregivers and broader supports. Patients' honest communication of symptoms to the clinicians treating them was also valued.

\section{Discordant perspectives}

Three main discordant perspectives between clinicians and consumers emerged from the data: divergent understanding of patient needs at end of life, divergent capacity of the 
healthcare setting/providers to accommodate family needs, and divergent knowledge and communication needs at end of life.

\section{Divergent understanding of patient needs at end of life}

“... She said she doesn't want to suffer... we talked about it more as a family and worked it out with the nurses and doctors here... I think it was very important to her that all of us were involved in the process." (family caregiver) [33].

Practitioners seemed to have a different way of offering comfort - to administer morphine through a syringe driver and this had the effect of sedating the person so that they were no longer aware of their surroundings [50].

With regard to the statement "An advance directive should always be followed", $88 \%$ of the relatives, $78 \%$ of the nurses and only 37\% of the physicians agreed [37].

Divergent attitudes between the person at the end of life, family carers and healthcare staff with regard to patient needs and their physical comfort and care needs were cited, for example symptom control [41, 50]. One article wrote that divergence occurred around the idea of what comfort provision was from the perspective of the healthcare provider: which was to achieve symptom control by administering medications including morphine. From the patients'/families' views in the study, such "comfort" meant the patient was sedated and unable to engage via their senses, spiritually or socially [50]. Another article confirmed the dissonance between nurses' perception that patients tolerated symptoms well and the patients' report that symptoms were distressing them [33]. Symptom identification tools (such as IPOS) gave patients the opportunity to highlight their most important concerns, which were overlooked previously [33]. Divergent preferences of the location of care at end of life were apparent. Whilst healthcare staff perceived that the acute hospital setting is inappropriate for end of life care when compared to continued care in the residential aged care setting, for many patients and their families the acute hospital setting was in fact seen to provide a "safe haven", hope of options to prolong life and a place of more comprehensive care [35].

Another common discordance related to patient needs at end of life was conflict over the use of advance care directives and decision capacity; conflict arose from healthcare staff's reluctance to enact advance care directives of patients with advance dementia [37, 51], with families generally advocating that the patient wishes should be followed. Another source of disagreement between these parties was about the healthcare providers' interpretation of and adherence to advance health directives such as withholding of antibiotics [51], or enabling euthanasia for people who previously requested but were now cognitively impaired [37].

\section{Divergent capacity of healthcare settings/providers to accommodate family needs}

Disagreement existed about the capacity of facility design priorities and healthcare provider roles to accommodate and be responsive to the needs of families in the EOL scenario $[40,49,50]$. The data suggested residential aged care facilities, especially those designed for care of people with dementia, prioritise design aspects that ease care delivery for staff and maximise resident safety without adequate consideration that these same spaces are a place of end-of-life care. Design aspects such as private spaces for relatives to grieve are absent [50]. Families described the experience of being "abandoned" on a ward with a loved one, feeling that staff were not "checking in" on the person at the end of life as some healthcare professionals were not aware of the patient and their family's need for ongoing clinical support, for example providing information about the dying process and delivering emotional support $[40,50]$. This contrasted with staff who noted their focus was to give space to families and believed they were enabling this by ceasing routine monitoring. These two scenarios highlighted the differing perspectives of healthcare professionals and families on the ideal scope of the clinician role [32, 49].

\section{Divergent knowledge and communication at end of life}

[Patient's] family were unaware of the "do not attempt cardio pulmonary resuscitation" order and would not have agreed to it. Staff members who were interviewed and spoke about this family described them as not being 'on the same page' as staff [40].

"There's a lot of people that will just sit and nod at a doctor and when they've gone, will ask the nurse." [40]

The final central discordance theme was the difference between what was being said by healthcare professionals and what is heard by families. The data suggested these distinct groups often bring different understandings to the end-of-life situation [35, 40, 42, 45, 51]. Data were reported that related to misunderstanding information and non-disclosure of information [40, 42]. One article detailed the experience of a family that thought their mother was being actively treated until her death, despite discussions with the healthcare team regarding her poor prognosis, when in fact 
healthcare staff had placed the patient on a care of the dying pathway. The patient had signed a "do not attempt cardiopulmonary resuscitation order" which the family stated they had not been made aware of [40]. Differing attitudes towards the end of life $[42,51]$ were captured in a nursing home between residents who were accepting of death, wanted to and were willing to speak about it and, the staff who noted they often withdrew from partaking in such discussions [51]. Conflicting expectations of active or palliative care $[35,44,45]$ were illustrated in an article exploring the difficulties influencing decision-making at the end of life for people with dementia [45]. This article described the view that relatives' expectations were shaped either by previous experience of dementia amongst people known to them or by occasions where their relatives' health had declined and then unexpectedly improved. This sometimes led to differences of opinion between family carers and healthcare practitioners, in terms of what each believes is in the best interests of the person with dementia. Practitioners explained, for example, that often families would want their relatives to be fed, even if they as clinicians felt this was not appropriate.

\section{Discussion}

Approaching the end of life in old age and from natural causes can be an orchestrated and comforting event when families, patients and clinicians have a common understanding of the irreversibility of the process and the goals of treatment. Alternatively it can be a traumatic event when perceptions of care are discordant between consumers and healthcare professional. Differences in perspective can result in family and patients feeling an overwhelming sense of abandonment as a reduction of monitoring, or minimisation of non-beneficial interventions are put in place by clinicians whose message has not been fully understood.

This scoping review summarised the concordant and discordant perceptions of care quality between the staff delivering and the consumers receiving end-of-life care. Patient, family and system issues related to communication arose in the concordance sphere, whereas misunderstanding on place and mode of care dominated the discordance domains. Comparing the concordant and discordant themes, further similarities and differences can be highlighted. In both domains, patient and family needs were strongly emphasised; healthcare providers and patients recognised that holistic patient-centred care and family involvement were important in the delivery of end-of-life care but contrasted in their views of what the patient and family needs were. Since the included studies were from a range of health systems around the world, a possible major explanation for these domain differences may be attributed to culture and country-specific operational factors, which may result in misinterpretation of needs. For example, families in Hong Kong were less inclined to discuss dying and preferred to persist with active treatment requests for their loved ones until death [32], whereas patients in England were supportive of advance care planning [31]; those in USA were more accepting of palliative care [51], more concerned with their own quality of life and more comfortable in raising the issue of too much treatment with doctors [47]. Cultural influences on the acceptance of death and willingness to refuse futile interventions have previously been identified by others and are known to hinder efforts to create barriers such as misunderstanding on the course of action between patients, families and healthcare providers; hindering attempts to initiate end-of-life discussions; reducing willingness to access certain health services; and accepting medications for symptom control that can potentially hasten death [53, 54].

These findings suggest that progress has been made in aligning patient's preference and healthcare providers' performance on delivery of good quality care that respects patient's wishes. Furthermore, clinicians' acceptance of family members as proactive advocates in the patient's best interest is improving as a better understanding of the critical role of family in supporting the dying, and the value of the partnership between providers and health service users has become apparent in recent years [32, 34]. Yet, gaps remain? the following: care coordination and open communication of prognosis, [31, 39, 46], public understanding of the meaning of goals of care as well as the associated management de-escalation, and finally the enactment of advance care directives by clinicians for people with diminished decision capacity $[37,51]$. The constraints of the environmental context and resourcing remain a barrier to the realisation of what healthcare professionals recognise is important in the delivery of good quality EOL, fully encompassing the needs of the patient and the family. A key area of concern outlined in the articles was the funding of aged care and the difficulty of securing enough resources (in a timely manner) to cope with patients' changing needs; for example, staff recognised the need to have more intensive one-to-one care when someone is dying, but staffing levels prevent this [50].

\section{Relevance to other literature}

Patients' and families' understanding of the need to prevent over-treatment through documentation of goals of care and preferences is essential for their experiencing satisfaction with EOL care such as receiving care aligned with preferences [55], timely hospice care [56], avoidance 
of unnecessary hospitalisation [57] and suffering and prolonged, futile intensive treatments [58]. Such understanding enables them to embrace the concept of a good death as part of the life cycle.

Consistent with our findings, support for emotional needs beyond the physical [59], avoidance of over-treatment [60] and enhancement of patient autonomy [61] in the last stages are promoted in several health systems to improve quality of care at the end of life. Our synthesis of studies published between 2000 and 2018 also revealed some less than optimal features affecting the perception of quality care at the end of life. Gaps in continuity of care for people with terminal illness and reductions in invasive treatments associated with continuity of care have been reported in quantitative studies for over a decade [62]. Even in more recent times, the reluctance to hold EOL conversations, and discomfort in engaging in them either with clinicians or among family members despite identified need continue to be reported [63], particularly in relation to patient preference for place of death and family readiness to take on that responsibility. This suggests the need for mass education strategies to normalise the conversation such as via social media channels [64] and information about alternative pathways alongside the potential burden on informal caregivers trying to honour patients' wishes. Further engagement of relevant staff in initiating the compassionate EOL discussions following targeted training has also been attempted [65], and while intention to change practice is reported by clinicians, hard evidence of actual impact is yet to be demonstrated.

\section{Strengths and limitations}

This review has a number of strengths. It was conducted by academics and clinicians from a variety of disciplines providing an interdisciplinary standpoint and included informal caregivers. The article search and selection were systematic with two authors involved in all stages, and the review captured published articles from a wide range of study designs, countries and from all key perspectives. The confirmation of end of life was supplemented with an objective and validated risk-of-death tool [27] if not clearly stated in the articles. Articles reflecting only one of the perspectives, i.e. clinicians-only or patient-/families-only viewpoints, are reported in separate manuscripts as these studies are different subcomponents of the umbrella project and cover other domains unrelated to discordance or concordance.

We also acknowledge that this study has some limitations. MEDLINE was the only database-accessed, and although it is the largest bibliographic data source in medicine ( $>26$ million records from $>5600$ sources), the lack of search in CINAHL, for instance, may have led to missed articles on the perspectives of allied health workers. While the 21 included studies were conducted in many different care settings across four continents and comprised both qualitative and quantitative designs, half of the studies were conducted in nursing homes including many patients with dementia, which may impact on the generalisability of findings. Satisfaction with and perceptions of good quality care at the end of life for people without cognitive impairment may not be affected by the same ethical and practical challenges of advance care planning for people with complex care needs and dementia; for instance, patient autonomy, ability for joint decision-making, documented goals of care, or offers of further active management rather than comfort care $[35,66]$. Due to resource constraints, we did not search the grey literature or conducted manual searches of reference lists from included studies, and given that this was a scoping review, we did not examine the risk of bias or other study quality parameters as the focus was to characterise the range of views from both providers and consumers.

\section{Implications for clinicians and future research}

Clinician awareness of the possibility of misconceptions by patients or anxious families is the first step in preventing discordance of views on care quality at the end of life. This can be addressed by open communication on prognosis (including disclosing the uncertainty around time to death) or disease trajectory and timely explanation of options including the shared decision on withdrawal of active treatment when appropriate and preferences around symptom management. While many efforts to document resuscitation plans and enhance the effectiveness of advance health directives are underway across health systems [67-69], there needs to be greater reinforcement of intensive public education on the appropriateness, indications and meaning of life-sustaining treatments and their difference with comfort care, especially in different cultural contexts, to alleviate current misunderstandings and perceptions of abandonment or malpractice. The clinical and cost-effectiveness of alternative models of palliative care service [6] that meet the local needs in areas with limited access [70] and impact of capacity building for staff working out of hospitals [71] are also worth investigating to offer real alternatives to patients preferring a noninstitutional death.

\section{Conclusion}

This review identified that consumers and healthcare professionals agree on several important aspects of treatments at the end-of-life care particularly related to respecting patient autonomy and the role of family. However, consumers and healthcare professionals have discordant views about the role of healthcare provider and the purpose of treatment withdrawal at the end of life. This conflict between consumers 
and healthcare professionals potentially hinders the value of advance care directives and impacts on trust in the health system to deliver care that meets patient expectations, whether or not well informed. Progress in delivering patientcentred care and acknowledging the role of families has become apparent in the literature. However, further efforts are needed in investigating ways to better communicate the goals of care, estimated time to death, and spelling out the consequences and limitations of treatment. A better public understanding of these concepts could prevent potentially harmful over-treatment and enhance satisfaction with care for dying individuals and their families.

Supplementary Information The online version contains supplementary material available at https://doi.org/10.1007/s41999-021-00549-6.

Acknowledgements We thank Dr Abela Mahimbo, Dr Mina Bakhit and Dr Loai Albarqouni for their advice on thematic process in the initial stages of this research and our university librarians for their assistance with interlibrary loans.

Author contributions MC, DB and ZAM performed concept, design, screening and full-text eligibility. JC done search strategy. DB and ZAM extracted the data. DB, ZAM, JC and ÉNS contributed to thematic analysis. JC, ZAM, MC, ÉNS and EP interpreted the data. MC, ZAM, ÉNS, JC and EP wrote the manuscript. All authors contributed to revisions.

Funding This research received no specific grant from any government, commercial, academic or not-for-profit organisations.

\section{Declarations}

Conflict of interest None to declare.

Ethics approval Not applicable. This article did not involve studies with human participants. Consent to participate not applicable.

\section{References}

1. Australian Commission on Safety and Quality in Health Care (2015) National Consensus Statement: essential elements for safe and high-quality end-of-life care. Australian Commission on Safety and Quality in Health Care, Sydney

2. Singer PA, Bowman KW (2002) Quality end-of-life care: a global perspective. BMC Palliat Care 1(1):4. https://doi.org/10.1186/ 1472-684X-1-4

3. Bloomer MJ, Hutchinson AM, Botti M (2019) End-of-life care in hospital: an audit of care against Australian national guidelines. Aust Health Rev 43(5):578-584. https://doi.org/10.1071/AH182 15

4. Clark K, Byfieldt N, Green M, Saul P, Lack J, Philips JL (2014) Dying in two acute hospitals: would usual care meet Australian national clinical standards? Aust Health Rev 38(2):223-229. https://doi.org/10.1071/ah13174

5. United Nations Department of Economic and Social AffairsPopulation Division (2017) World Population Ageing_-Highlights (ST/ESA/SER.A/397), Division P
6. Chapman M, Johnston N, Lovell C, Forbat L, Liu WM (2018) Avoiding costly hospitalisation at end of life: findings from a specialist palliative care pilot in residential care for older adults. BMJ Support Palliat Care 8(1):102-109. https://doi.org/10.1136/bmjsp care-2015-001071

7. Ali M, Capel M, Jones G, Gazi T (2019) The importance of identifying preferred place of death. BMJ Support Palliat Care 9(1):84. https://doi.org/10.1136/bmjspcare-2015-000878

8. Beernaert K, Van den Block L, Van Thienen K, Devroey D, Pardon K, Deliens L et al (2015) Family physicians' role in palliative care throughout the care continuum: stakeholder perspectives. Fam Pract 32(6):694-700. https://doi.org/10.1093/fampra/cmv072

9. Singer PA, Martin DK, Kelner M (1999) Quality end-of-life carepatients' perspectives. JAMA 281(2):163-168. https://doi.org/10. 1001/jama.281.2.163

10. Galushko M, Romotzky V, Voltz R (2012) Challenges in end-oflife communication. Curr Opin Support Palliat Care 6(3):355364. https://doi.org/10.1097/SPC.0b013e328356ab72

11. White DB, Ernecoff N, Buddadhumaruk P, Hong S, Weissfeld L, Curtis JR et al (2016) Prevalence of and factors related to discordance about prognosis between physicians and surrogate decision makers of critically ill patients. JAMA 315(19):2086-2094. https://doi.org/10.1001/jama.2016.5351

12. Fried TR, Bradley EH, O'Leary J (2003) Prognosis communication in serious illness: perceptions of older patients, caregivers, and clinicians. J Am Geriatr Soc 51(10):1398-1403. https://doi. org/10.1046/j.1532-5415.2003.51457.x

13. Gott M, Ingleton C, Gardiner C (2013) Chapter 3, Exploring the transition from curative care to palliative care: a systematic review of the literature (phase 1). Transitions to palliative care for older people in acute hospitals: a mixed-methods study. Health Services and Delivery Research, vol 1.11. NIHR Journals Library, Southampton

14. Downar J, Delaney JW, Hawryluck L, Kenny L (2016) Guidelines for the withdrawal of life-sustaining measures. Intensive Care Med 42(6):1003-1017. https://doi.org/10.1007/s00134-016-4330-7

15. Davies C, Fattori F, O’Donnell D, Donnelly S, Ní Shé É, Shea MO et al (2019) What are the mechanisms that support healthcare professionals to adopt assisted decision-making practice? A rapid realist review. BMC Health Serv Res 19(1):960. https://doi.org/ 10.1186/s12913-019-4802-x

16. Manalo MFC (2013) End-of-life decisions about withholding or withdrawing therapy: medical, ethical, and religio-cultural considerations. Palliat Care 7:1-5. https://doi.org/10.4137/PCRT.S10796

17. Ní Shé É, O’Donnell D, Donnelly S, Davies C, Fattori F, Kroll $\mathrm{T}$ (2020) "What bothers me most is the disparity between the choices that people have or don't have": a qualitative study on the health systems responsiveness to implementing the assisted decision-making (Capacity) Act in Ireland. Int J Environ Res Public Health. https://doi.org/10.3390/ijerph17093294

18. Cardona M, Shanmugam S, Lewis ET, Psirides A, Anstey M, Hillman K (2019) A rapid review of patient and family perspectives on inappropriateness of intensive care treatments at the end of life. medRxiv. https://doi.org/10.1101/19007138

19. Willmott L, White B, Smith MK, Wilkinson DJ (2014) Withholding and withdrawing life-sustaining treatment in a patient's best interests: Australian judicial deliberations. Med J Aust 201(9):545-547. https://doi.org/10.5694/mja13.10874

20. Krawczyk M, Gallagher R (2016) Communicating prognostic uncertainty in potential end-of-life contexts: experiences of family members. BMC Palliat Care 15(1):59. https://doi.org/10.1186/ s12904-016-0133-4

21. de Boer D, Hofstede JM, de Veer AJE, Raijmakers NJH, Francke AL (2017) Relatives' perceived quality of palliative care: comparisons between care settings in which patients die. BMC Palliat Care 16(1):41. https://doi.org/10.1186/s12904-017-0224-x 
22. Lapid MI, Koopmans R, Sampson EL, Van den Block L, Peisah C (2020) Providing quality end-of-life care to older people in the era of COVID-19: perspectives from five countries. Int Psychogeriatr. https://doi.org/10.1017/S1041610220000836

23. Emanuel EJ, Persad G, Upshur R, Thome B, Parker M, Glickman A et al (2020) Fair allocation of scarce medical resources in the time of Covid-19. N Engl J Med 382(21):2049-2055. https://doi. org/10.1056/NEJMsb2005114

24. Tong A, Flemming K, McInnes E, Oliver S, Craig J (2012) Enhancing transparency in reporting the synthesis of qualitative research: ENTREQ. BMC Med Res Methodol 12(1):181. https:// doi.org/10.1186/1471-2288-12-181

25. Martin GP, Jenkins DA, Bull L, Sisk R, Lin L, Hulme W et al (2020) Toward a framework for the design, implementation, and reporting of methodology scoping reviews. J Clin Epidemiol 127:191-197. https://doi.org/10.1016/j.jclinepi.2020.07.014

26. Bannach-Brown A, Clark J (2018) Systematic review \& metaanalysis: automation tools to help your review. Bond University, Gold Coast, QLD. https://osf.io/8fsry/. Accessed May 2020

27. Cardona M, Lewis ET, Kristensen MR, Skjot-Arkil H, Ekmann AA, Nygaard HH et al (2018) Predictive validity of the CriSTAL tool for short-term mortality in older people presenting at Emergency Departments: a prospective study. Eur Geriatric Med 9(6):891-901. https://doi.org/10.1007/s41999-018-0123-6

28. Nili A, Tate M, Barros A, Johnstone D (2020) An approach for selecting and using a method of inter-coder reliability in information management research. Int J Inf Manage 54:102154. https:// doi.org/10.1016/j.ijinfomgt.2020.102154

29. Thomas J, Harden A (2008) Methods for the thematic synthesis of qualitative research in systematic reviews. BMC Med Res Methodol 8(1):45. https://doi.org/10.1186/1471-2288-8-45

30. Feldman R, Berman N, Reid MC, Roberts J, Shengelia R, Christianer K et al (2013) Hemodialysis patients: identifying barriers and future directions. J Palliat Med 16(12):1528-1533

31. Hall S, Goddard C, Stewart F, Higginson IJ (2011) Implementing a quality improvement programme in palliative care in care homes: a qualitative study. BMC Geriatr 11(1):31. https://doi.org/ 10.1186/1471-2318-11-31

32. Ho AH, Luk JK, Chan FH, Chun Ng W, Kwok CK, Yuen JH et al (2016) Dignified palliative long-term care: an interpretive systemic framework of end-of-life integrated care pathway for terminally ill Chinese older adults. Am J Hosp Palliat Care 33(5):439-447. https://doi.org/10.1177/1049909114565789

33. Kane PM, Ellis-Smith CI, Daveson BA, Ryan K, Mahon NG, McAdam B et al (2018) Understanding how a palliative-specific patient-reported outcome intervention works to facilitate patientcentred care in advanced heart failure: a qualitative study. Palliat Med 32(1):143-155. https://doi.org/10.1177/0269216317738161

34. Östlund U, Blomberg K, Söderman A, Werkander HC (2019) How to conserve dignity in palliative care: suggestions from older patients, significant others, and healthcare professionals in Swedish municipal care. BMC Palliat Care 18(1):10. https://doi.org/10. 1186/s12904-019-0393-x

35. Reyniers T, Houttekier D, Cohen J, Pasman HR, Deliens L (2014) The acute hospital setting as a place of death and final care: a qualitative study on perspectives of family physicians, nurses and family carers. Health Place 27:77-83. https://doi.org/10.1016/j. healthplace.2014.02.002

36. Rich SE, Williams CS, Zimmerman S (2010) Concordance of family and staff member reports about end of life in assisted living and nursing homes. Gerontologist 50(1):112-120. https://doi.org/ 10.1093/geront/gnp089

37. Rurup ML, Onwuteaka-Philipsen BD, Pasman HR, Ribbe MW, van der Wal G (2006) Attitudes of physicians, nurses and relatives towards end-of-life decisions concerning nursing home patients with dementia. Patient Educ Couns 61(3):372-380. https://doi. org/10.1016/j.pec.2005.04.016

38. Kaasalainen S, Ploeg J, McAiney C, Schindel Martin L, Donald F, Martin-Misener R et al (2013) Role of the nurse practitioner in providing palliative care in long-term care homes. Int $\mathrm{J}$ Palliat Nurs 19(10):477-485. https://doi.org/10.12968/ijpn.2013.19.10. 477

39. Browne S, Macdonald S, May CR, Macleod U, Mair FS (2014) Patient, carer and professional perspectives on barriers and facilitators to quality care in advanced heart failure. PLoS One 9(3):e93288. https://doi.org/10.1371/journal.pone.0093288

40. Caswell G, Pollock K, Harwood R, Porock D (2015) Communication between family carers and health professionals about end-of-life care for older people in the acute hospital setting: a qualitative study. BMC Palliat Care 14(1):35. https://doi.org/10. 1186/s12904-015-0032-0

41. Chan J, Kayser-Jones J (2005) The experience of dying for Chinese nursing home residents: cultural considerations. J Gerontol Nurs 31(8):26-32. https://doi.org/10.3928/0098-9134-2005080111 (quiz 52-3)

42. Costello $J$ (2001) Nursing older dying patients: findings from an ethnographic study of death and dying in elderly care wards. J Adv Nurs 35(1):59-68. https://doi.org/10.1046/j.1365-2648.2001. 01822.x

43. Dening KH, Greenish W, Jones L, Mandal U, Sampson EL (2012) Barriers to providing end-of-life care for people with dementia: a whole-system qualitative study. BMJ Support Palliat Care 2(2):103-107. https://doi.org/10.1136/bmjspcare-2011-000178

44. Johnston B, Patterson A, Bird L, Wilson E, Almack K, Mathews $\mathrm{G}$ et al (2018) Impact of the Macmillan specialist Care at Home service: a mixed methods evaluation across six sites. BMC Palliat Care 17(1):36. https://doi.org/10.1186/s12904-018-0281-9

45. Lamahewa K, Mathew R, Iliffe S, Wilcock J, Manthorpe J, Sampson EL et al (2018) A qualitative study exploring the difficulties influencing decision making at the end of life for people with dementia. Health Expect 21(1):118-127. https://doi.org/10.1111/ hex. 12593

46. Slatyer S, Toye C, Popescu A, Young J, Matthews A, Hill A et al (2013) Early re-presentation to hospital after discharge from an acute medical unit: perspectives of older patients, their family caregivers and health professionals. J Clin Nurs 22(3-4):445-455. https://doi.org/10.1111/jocn.12029

47. Steinhauser KE, Clipp EC, McNeilly M, Christakis NA, McIntyre LM, Tulsky JA (2000) In search of a good death: observations of patients, families, and providers. Ann Intern Med 132(10):825-832. https://doi.org/10.7326/0003-4819-132-10200005160-00011

48. van de Pol MH, Fluit CR, Lagro J, Niessen D, Rikkert MG, LagroJanssen AL (2015) Quality care provision for older people: an interview study with patients and primary healthcare professionals. Br J General Pract 65(637):e500-e507. https://doi.org/10. 3399/bjgp15X686065

49. Brogaard T, Jensen AB, Sokolowski I, Olesen F, Neergaard MA (2011) Who is the key worker in palliative home care? Scand J Prim Health Care 29(3):150-156. https://doi.org/10.3109/02813 432.2011.603282

50. Fleming R, Kelly F, Stillfried G (2015) "I want to feel at home": establishing what aspects of environmental design are important to people with dementia nearing the end of life. BMC Palliat Care 14:26. https://doi.org/10.1186/s12904-015-0026-y

51. Forbes $\mathrm{S}$ (2001) This is Heaven's waiting room: end of life in one nursing home. J Gerontol Nurs 27(11):37-45. https://doi.org/10. 3928/0098-9134-20011101-10 
52. Institute of Medicine (US) Committee on Quality of Health Care in America (2001) Crossing the quality Chasm: a newhealth system for the 21st century. Institute of Medicine, Washington

53. Givler A, Bhatt H, Maani-Fogelman PA (2020) The Importance of Cultural Competence in Pain and Palliative Care. StatPearls [Internet]. StatPearls Publishing, Treasure Island

54. Clark K, Phillips J (2010) End of life care-the importance of culture and ethnicity. Aust Fam Physician 39(4):210-213

55. Mack JW, Weeks JC, Wright AA, Block SD, Prigerson HG (2010) End-of-Life discussions, goal attainment, and distress at the end of life: predictors and outcomes of receipt of care consistent with preferences. J Clin Oncol 28(7):1203-1208. https://doi.org/10. 1200/JCO.2009.25.4672

56. Bischoff KE, Sudore R, Miao Y, Boscardin WJ, Smith AK (2013) Advance care planning and the quality of end-of-life care in older adults. J Am Geriatr Soc 61(2):209-214. https://doi.org/10.1111/ jgs. 12105

57. Orlovic M, Callender T, Riley J, Darzi A, Droney J (2020) Impact of advance care planning on dying in hospital: evidence from urgent care records. PLoS One 15(12):e0242914. https://doi.org/ 10.1371/journal.pone.0242914

58. Salama H, Al Mutairi N, Damlaj M, Alolayan AAM, Binahmed A, Salama $\mathrm{H}$ et al (2020) Reducing futile acute care services (ACS) for terminally ill cancer patients (Dignity Project). J Clin Oncol 38(29_suppl):23. https://doi.org/10.1200/JCO.2020.38.29_suppl. 23

59. Lee E, Sussman T, Kaasalainen S, Durepos P, McCleary L, Wickson-Griffiths A et al (2020) The relationship between caregivers' perceptions of end-of-life care in long-term care and a good resident death. Palliat Support Care. https://doi.org/10.1017/S1478 951520000292

60. Thomas K, Gray SM (2018) Population-based, person-centred end-of-life care: time for a rethink. Br J General Pract 68(668):116-117. https://doi.org/10.3399/bjgp18X694925

61. Galekop MMJ, van Dijk HM, van Exel J, Cramm JM (2019) Views of professionals and volunteers in palliative care on patient-centred care: a Q-methodology study in the Netherlands. BMC Palliat Care 18(1):97. https://doi.org/10.1186/s12904-019-0479-5

62. Chen AY, Chen B, Kuo C-C (2020) Better continuity of care improves the quality of end-of-life care among elderly patients with end-stage renal disease. Sci Rep 10(1):19716. https://doi. org/10.1038/s41598-020-76707-w
63. Im J, Mak S, Upshur R, Steinberg L, Kuluski K (2019) "Whatever happens, happens" challenges of end-of-life communication from the perspective of older adults and family caregivers: a Qualitative study. BMC Palliat Care 18(1):113. https://doi.org/10.1186/ s12904-019-0493-7

64. MacKenzie AR, Lasota M (2020) Bringing Life to death: the need for honest, compassionate, and effective end-of-life conversations. Am Soc Clin Oncol Educ Book 40:476-484. https://doi.org/10. 1200/EDBK_279767

65. Rawlings D, Devery K, Poole N (2019) Improving quality in hospital end-of-life care: honest communication, compassion and empathy. BMJ Open Qual. 8(2):e000669-e. https://doi.org/10. 1136/bmjoq-2019-000669

66. Jones K, Birchley G, Huxtable R, Clare L, Walter T, Dixon J (2016) End of life care: a scoping review of experiences of advance care planning for people with dementia. Dementia 18(3):825-845. https://doi.org/10.1177/1471301216676121

67. Sittisombut S, Maxwell C, Love EJ, Sitthi-Amorn C (2008) Effectiveness of advance directives for the care of terminally ill patients in Chiang Mai University Hospital, Thailand. Nurs Health Sci 10(1):37-42. https://doi.org/10.1111/j.1442-2018.2007.00371.x

68. Butler M, Ratner E, McCreedy E, Shippee N, Kane RL (2014) Decision aids for advance care planning: an overview of the state of the science. Ann Intern Med 161(6):408-418. https://doi.org/ 10.7326/m14-0644

69. Pearce J, Ridley J (2016) Communicating in life-limiting illness: a practical guide for physicians. BC Med J 58(5):262-267

70. Wenham S, Cumming M, Saurman E (2020) Improving palliative and end-of-life care for rural and remote Australians. Public Health Res Pract. https://doi.org/10.17061/phrp3012001

71. Kaasalainen S, Sussman T, McCleary L, Thompson G, Hunter PV, Wickson-Griffiths A et al (2019) Palliative care models in longterm care: a scoping review. Nurs Leadersh (Tor Ont) 32(3):8-26. https://doi.org/10.12927/cjnl.2019.25975

Publisher's Note Springer Nature remains neutral with regard to jurisdictional claims in published maps and institutional affiliations. 